\title{
Madurez psicológica y bienestar psicológico en estudiantes de una Institución Educativa Privada de Juliaca - Perú
}

\section{Psychological maturity and psychological well-being in students of a Private Educational Institution in Juliaca - Peru}

\author{
Yesenia Mamani Sucto1, Yessika Mamani-Quispe², Eddy Aquize Anco³
}

\begin{abstract}
RESUMEN
Objetivo: Determinar la relación entre madurez psicológica y bienestar psicológico en estudiantes de una Institución Educativa Privada de Juliaca. Metodología: Estudio correlacional, de diseño no experimental y corte transversal, aplicado sobre una población de 78 estudiantes de ambos sexos del cuarto y quinto grado de educación secundaria; a quienes se les aplicó la escala de Bienestar Psicológico de Ryff y el cuestionario de Madurez Psicológica (PSYMAS). La encuesta se realizó de manera online y los análisis consideraron un nivel de significancia del $5 \%$. Resultados: Un $61.5 \%$ de los encuestados evidencia un nivel moderado de madurez psicológico, y, el 100\% evidencia bienestar a nivel bajo. El análisis inferencial demostró correlaciones significativas entre la madurez psicológico y el bienestar psicológico (Rho $=.289, \mathrm{p}<.05)$. Conclusión: En la medida que los adolescentes sean maduros psicológicamente, entonces, más probabilidad existe tener sensaciones placenteras a lo largo de la vida o satisfacer el deseo personal.
\end{abstract}

Palabras clave: Bienestar psicológico, madurez psicológica, adolescencia

\begin{abstract}
Objective: To determine the relationship between psychological maturity and psychological well-being in students of a Private Educational Institution in Juliaca. Methodology: A correlational study, with a non-experimental design and a cross-sectional section, applied to a population of 78 students of both sexes in the fourth and fifth grade of secondary education; to whom the Ryff Psychological Wellbeing scale and the Psychological Maturity questionnaire (PSYMAS) were applied. The survey was conducted online and the analyzes considered a significance level of $5 \%$. Results: $61.5 \%$ of those surveyed show a moderate level of psychological maturity, and $100 \%$ show well-being at a low level. Inferential analysis showed significant correlations between psychological maturity and psychological well-being $(\mathrm{Rho}=.289, \mathrm{p}<.05)$. Conclusion: To the extent that adolescents are psychologically mature, then, the more likely it is to have pleasant sensations throughout life or to satisfy personal desire.
\end{abstract}

Keywords: Psychological well-being, psychological maturity, adolescence.

\footnotetext{
${ }^{1}$ Universidad Peruana Unión, Juliaca, Perú Orcid ID: 0000-0001-8871-5067

${ }^{2}$ Institución Educativa Privada "SIGMA", Juliaca, Perú

Orcid ID: 0000-0002-4469-4273

3Universidad Peruana Unión, Juliaca, Perú

Orcid ID: 0000-0002-4820-9570
} 


\section{INTRODUCCIÓN}

En los últimos años la investigación en el ámbito de la psicología se ha centrado en la salud mental de los adolescentes (Perales et al., 2019); puesto que problemas en este ámbito repercuten en su desarrollo normal en distintos ámbitos como el personal, social, educativo y familiar (Navarro-Loli et al., 2017).

En el Perú, se estima que uno de cada cinco niños y adolescentes tiene alguna vulnerabilidad de salud mental, lo cual indica una gran dificultad en el bienestar emocional requiriendo de ayuda inmediata, además, se reporta que el problema más frecuente en este ámbito tiene que ver con la aparición de depresión, ideas suicidas y otros trastornos emocionales (Diario Peru21, 2018). Es en tal contexto que el Ministerio de la Salud refiere que gozar de buena salud mental es equivalente a tener un estado de equilibrio psicológico (Ministerio de Salud, 2017).

Es en esta etapa que el individuo aprende a establecer su independencia emocional y psicológica, percibiendo tener un derecho a la información, educación, salud y justicia, a un entorno seguro, además de, poder adquirir nuevos conocimientos y disponer de oportunidades para expresar sus opiniones, ya que en la actualidad experimentan diferentes estados de ánimo con los cuales deben de lidiar, por ello cada decisión que tomen tiene consecuencias permanentes en su vida (Aláez et al., 2003).

Es en este escenario que surge la importancia de estudiar algunas variables importantes para la etapa adolescente. Una de ellas es la madurez psicológica, un concepto abstracto que generalmente se utiliza para describir la seguridad que un individuo tiene para enfrentarse al día a día, demostrando tener tranquilidad a la hora de afrontar adversidades y situaciones conflictivas (Zacarés \& Serra, 1996). Por ello, desarrollar esta capacidad permite al adolescente percibir la importancia de sus decisiones y ser consciente de las posibles consecuencias de sus propias acciones (Rodrigo \& Andreu, 2017). Al respecto, es bien sabido que los adolescentes pueden tomar decisiones equivocadas, ya que no distinguen de manera adecuada los riesgos y consecuencias; de este modo, son vulnerables a la influencia de grupo y al entorno que lo rodea por lo que pueden incurrir en conductas de riesgo (García et al., 2015).
Otra de las variables a incluir en la discusión es el bienestar psicológico, el cual es un constructo amplio que incluye dimensiones sociales, subjetivas y psicológicas, y se evidencia a través de comportamientos relacionados con la salud que llevan a las personas a tener un modo de pensar y sentirse aliviado y positivo, lo cual genera un adecuado manejo de su estado emocional (Freire, 2014). Por su parte, Carol Ryff, autora del constructo refiere que es un conjunto de sensaciones relacionadas a experimentar situaciones placenteras a lo largo de la vida o satisfacer el deseo personal (Gaxiola-Villa, 2014).

La relación entre la madurez psicológica y el bienestar psicológico ha sido documentada en algunasinvestigaciones reportadas enlaliteratura científica internacional; así, se reconoce que en la medida que un adolescente demuestre tener un adecuado bienestar psicológico, entonces existe probabilidad de tener un mejor ajuste adecuado de ideas, pensamientos y acciones (Joy \& Mathew, 2018). El hecho de gozar de sensaciones plancenteras fruto de un estilo de vida que implica sentirse bien y con salud mental, promueve comportamientos maduros ajustados a la realidad y etapa del individuo (McCrae, 2002). Sobre todo, en el ámbito social, que es un aspecto en muchos casos percibido como problema para los adolescentes con déficit de habilidades, o, con problemas para desenvolverse en su contexto familiar o social; situación distinta a la de adolescentes con madurez psicológica, que debido a su bienestar psicológico pueden afrontar todo tipo de situaciones sociales conflictivas y adversas (Dewi et al., 2018; Zacarés \& Serra, 1996).

En función de lo expuesto, el objetivo de la presente investigación es analizar la relación entre la madurez psicológica y el bienestar psicológico en estudiantes secundarios de una Institución Educativa Privada de Juliaca.

\section{METODOLOGÍA}

Corresponde a un estudio de tipo correlacional porque se pretende medir el grado de asociación entre dos variables, de diseño no experimental porque no se pretende manipular la variable independiente, y de corte transversal por que se levantaron los datos en un solo momento en la línea del tiempo (Hernandez et al., 2014). 


\section{Participantes}

La población estuvo conformada por la totalidad de estudiantes (78) de ambos sexos del cuarto y quinto grado de secundaria de la Institución Educativa Privada Sigma de la ciudad de Juliaca. En este caso no fue necesario realizar un muestreo debido a que la cantidad total fue accesible y alcanzable para aplicar la encuesta.

\section{Instrumentos}

Escala de Bienestar psicológico de Ryff. Este instrumento fue creado por Carol Ryff en el año 1989 y adaptado al español por Díaz et al. (2006). Cuenta con 39 ítems distribuidos en seis dimensiones: Auto aceptación, Relaciones positivas, Autonomía, Dominio del entorno, Crecimiento personal y Propósito de vida. Las opciones de respuesta tienen formato tipo Likert y se puntúan de la siguiente manera: 1= Totalmente en desacuerdo, 2= En desacuerdo, 3=Ni de acuerdo ni desacuerdo, 4= De acuerdo, $5=$ Totalmente de acuerdo. Las propiedades psicométricas que se hallaron para la presente investigación, fueron de un $\alpha=, 784$ el cual indica un índice aceptable por otro lado, se halló el índice de validez de contenido el cual se extrajo a partir de cuatro expertos, los resultados indican un $\mathrm{V}=, 949$.

Cuestionario de Madurez Psicológica (PSYMAS, por sus siglas en ingles). Este instrumento fue elaborado por Morales, Camps y Lorenzo (2010, citado en Arana, 2014) y adaptado al Perú por Vera (2016). Está compuesto por 26 ítems distribuidos en tres dimensiones: Orientación al trabajo, Autonomía e Identidad; además, las opciones de respuesta son de tipo Likert y se puntúan de la siguiente manera: 1= Completamente en desacuerdo, 2= Bastante en desacuerdo, 3=Ni de acuerdo ni desacuerdo, 4= Bastante de acuerdo, 5=Completamente de acuerdo. La confiabilidad en la presente investigación se halló mediante el coeficiente Alpha de Cronbach, obteniendo un valor de .771 , el cual indica un valor aceptable. Por otra parte, en cuanto a la validez de contenido, este halló mediante el criterio de jueces calculando un $\mathrm{V}$ de Aiken de .971, que es un indicador de nivel alto.

\section{Procesamiento de datos}

Para los análisis se utilizó el programa estadístico SPSS versión 25 y se realizó por etapas. En la primera etapa se analizó las características demográficas de la población. En la segunda etapa, se analizó las frecuencias y porcentajes en niveles de las variables de estudio, así también, se analizó si los datos siguen o no una distribución normal. En la tercera etapa, se realizó un análisis de correlación mediante el estadístico Rho de Spearman entre las variables de estudio.

\section{RESULTADOS}

\section{Resultados del análisis sociodemográfico}

Se observa en la tabla 1 los resultados del análisis sociodemográfico aplicado a la población de estudio. En cuanto a la variable edad se observa que $48.7 \%$ tiene 16 años, seguido por un $44.9 \%$ que tiene 15 años, y finalmente, un $6.4 \%$ tiene 17 años. En cuanto a la variable sexo, existe mayor proporción de mujeres $(61.5 \%)$ que varones (38.5\%). En la misma línea, se observa que $57.7 \%$ pertenece al quinto grado y un $42.3 \%$ al cuarto grado. Finalmente, $84.6 \%$ son de la sierra, $9.0 \%$ de la selva y $6.4 \%$ de la costa.

Tabla 1

Características demográficas de los participantes

\begin{tabular}{llll} 
Variable & Categoría & N & $\%$ \\
Edad & 15 años & 35 & 44.9 \\
& 16 años & 38 & 48.7 \\
& 17 años & 5 & 6.4 \\
& Total & 78 & 100.0 \\
Sexo & Femenino & 48 & 61.5 \\
& Masculino & 30 & 38.5 \\
Grado & Total & 78 & 100.0 \\
& Cuarto & 33 & 42.3 \\
& Quinto & 45 & 57.7 \\
Procedencia & Cotal & 78 & 100.0 \\
& Sierra & 5 & 6.4 \\
& Selva & 7 & 84.6 \\
& Total & 78 & 9.0 \\
\hline
\end{tabular}




\section{Resultados descriptivos}

Se observa en la tabla 2 los resultados del análisis descriptivo realizado a las variables de estudio. Resalta el hecho de que un $61.5 \%$ de encuestados evidencia un nivel medio de madurez psicológica, seguido por un $38.5 \%$ de quienes evidencian un nivel bajo, mientras que ninguno evidencia un nivel alto. Por otra parte, en cuanto al bienestar psicológico, el $100 \%$ de los encuestados revela un nivel bajo, ninguno percibe estar en un nivel medio ni alto.

Tabla 2

Niveles de madurez psicológico y bienestar psicológico

\begin{tabular}{llll} 
Variable & Categoría & $\mathrm{N}$ & $\%$ \\
Madurez psicológica & Bajo & 30 & 38.5 \\
& Medio & 48 & 61.5 \\
& Alto & 0 & 0.0 \\
& Total & 78 & 100.0 \\
\hline Bienestar psicológico & Bajo & 78 & 100.0 \\
& Medio & 0 & 0.0 \\
& Alto & 0 & 0.0 \\
& Total & 78 & 100.0 \\
\hline
\end{tabular}

\section{Resultados inferenciales}

Por lo observado en la tabla 3, se sometió a análisis los puntajes obtenidos por la población de estudio, bajo la técnica de Kolmogorov-Smirnov (ideal para casos mayores a 30), obteniendo valores de $p$ de .200 para la variable bienestar psicológico y .001 para la variable madurez psicológica. Al respecto, debido que solo uno de los valores sobrepasa el .05, entonces, se acepta la Ha en ambos casos, suponiendo que los datos no provienen de una distribución normal. Esto tiene implicancias en el estadístico de prueba, el cual deberá ser no paramétrico.

Tabla 3

Análisis de normalidad

\begin{tabular}{llll} 
& & Bienestar psicológico & Madurez psicológica \\
\hline N & & 78 & 78 \\
& Media & 116,4231 & 82,3077 \\
Máximas diferencias extremas & Desviación estándar & 10,40295 & 10,47360 \\
& Absoluta &, 061 &, 142 \\
& Positivo &, 047 &, 113 \\
Estadístico de prueba & Negativo &,- 061 &,- 142 \\
Sig. asintótica (bilateral) & &, 061 &, 142 \\
\hline
\end{tabular}
a. La distribución de prueba es normal.
b. Se calcula a partir de datos.
c. Corrección de significación de Lilliefors.
d. Esto es un límite inferior de la significación verdadera. 
A continuación, en la tabla 4 se observan los resultados del análisis de correlación mediante el estadístico de prueba Rho de Spearman. En tal sentido, se evidencian correlaciones significativas entre la escala total de madurez psicológica y bienestar psicológica ( $\operatorname{Rho}=.289, p<.05$ ), de igual forma entre la madurez psicológica y las dimensiones de bienestar: auto aceptación $(p<.05)$, dominio del entorno $(p<.05)$, crecimiento personal $(p<.05)$ y propósito de vida $(p<.05)$. Mientras que se observaron correlaciones altamente significativas entre bienestar psicológico y las dimensiones de madurez: orientación al trabajo $(p<.01)$, autonomía $(p<.01)$ e identidad $(p<.01)$. Por último, no se hallaron correlaciones significativas entre la variable madurez y las dimensiones de bienestar: relaciones positivas $(p=.051)$ y autonomía $(p=.155)$.

Tabla 4

Análisis de correlación entre las variables de estudio

\begin{tabular}{lll} 
Variable & Bienestar psicológico & \\
\cline { 2 - 3 } Madurez psicológica & Rho & $\mathrm{P}$ \\
Orientación al trabajo & $.289^{*}$ & .010 \\
Autonomía & $.332^{* *}$ & .003 \\
Identidad & $.723^{* *}$ & .000 \\
\hline Variable & $.799^{* *}$ & .000 \\
\hline Bienestar psicológico & Madurez psicológica & \\
Auto aceptación & $.289^{*}$ & .010 \\
Relaciones positivas & $.260^{*}$ & .021 \\
Autonomía & .051 & .654 \\
Dominio del entorno & .155 & .176 \\
Crecimiento personal & $.240^{*}$ & .035 \\
Propósito de vida & $.282^{*}$ & .012 \\
\hline
\end{tabular}

* La correlación es significativa al .05

**La correlación es significativa al .01

\section{DISCUSIÓN}

La literatura científica, así como la comunidad de investigadores y especialistas en salud, resaltan la importancia de estudiar la salud mental de los adolescentes. Una razón principal tiene que ver con el hecho de prevenir trastornos psicológicos que en la etapa adulta puede desencadenar otros problemas más graves de conducta 0 desequilibrio emocional ante la familia y la sociedad. Es por ello que el objetivo de la presente investigación fue analizar la relación entre la madurez psicológica y el bienestar psicológico en estudiantes secundarios de una Institución Educativa Privada de Juliaca.

En cuanto a la hipótesis general, el análisis de correlación demuestra que existe una relación directa y significativa entre las variables bienestar psicológico y madurez psicológica $($ Rho $=.289, p<.05)$. Este hallazgo corrobora los resultados encontrados por Fossas (2019) quien estudió si la madurez psicológica puede predecir diferentes formas de felicidad, dentro de ellas el aspecto del bienestar psicológico, encontrando que, indicadores de felicidad $y$ bienestar pueden cambiar a medida que un adulto madura psicológicamente; por lo tanto, existe una relación teórica directa. En cuanto a una explicación teórica sobre estos hechos, una principal recae sobre la teoría de bienestar de la psicología positiva que implica asumir que la madurez, que viene a ser la capacidad para responder al medio ambiente, genera las condiciones psicológicas para percibir determinado nivel de bienestar en el individuo (Garcia-Landete, 2012).

En cuanto a las hipótesis específicas, el análisis de correlación demuestra la existencia de correlaciones directas entre las dimensiones de auto aceptación (Rho $=.260)$, dominio del 
entorno (Rho $=.240$ ), crecimiento personal (Rho $=.282)$, propósito de vida (Rho $=.264)$; por otra parte, no se evidenciaron correlaciones con las dimensiones Relaciones positivas $(p>.05)$ y autonomía $(p>.05)$. En relación a las correlaciones halladas, estos resultados guardan relación con otros hallazgos reportados en la literatura científica; por ejemplo, GonzálezFuentes y Andrade (2013) hallaron que la auto aceptación era un factor de riesgo para el intento suicida, no obstante, el nivel de auto aceptación dependía del grado de madurez psicológica alcanzado; seguidamente, Cortés et al. (2017) hallaron que el crecimiento personal dependía en gran manera de un componente psicológico vital como es la madurez alcanzada emocionalmente. Por otra parte, Páramo et al. (2012) refiere que los objetivos de vida en los adolescentes en etapa estudiantil son influenciados hasta cierto punto por el bienestar y la capacidad de hacer frente a los problemas, este último, producto de una madurez emocional.

Un aspecto importante a discutir es el hecho de no haber hallado correlaciones con las dimensiones relaciones positivas y autonomía; componentes que según Kern et al. (2015) hacen que el individuo se sienta integrado en la sociedad y lograr estar satisfecho con sus relaciones sociales. Sin embargo, una de las razones para no detectar relaciones significativas podría ser el hecho de encontrar limitaciones en la presente investigación, donde, la principal es el tamaño de población que es menor a 100 casos y no permite una mayor generalización.

\section{Declaración de financiamiento y de conflictos de interés:}

El estudio fue financiado por los autores, quienes declaran no tener conflictos de interés.

\section{Correspondencia}

Yesenia Mamani Sucto

Correo electrónico:

yesi.maps.you@gmail.com

Yessika Mamani-Quispe

Correo electrónico:

yessika.mq@gmail.com
Eddy Aquize Anco

Correo electrónico:

eddy.wildmar@upeu.edu.pe

\section{REFERENCIAS BIBLIOGRÁFICAS}

Aláez, M., Madrid, J., \& Antona, A. (2003). Adolescencia y salud. Papeles Del Psicólogo, 23(84), 45-53. http://www.redalyc.org/articulo. oa? id=77808405

Arana, D. (2014). Propiedades psicométricas del cuestionario de madurez psicològica en adolescentes del distrito de Casa Grande. Revista de Psicología de Trujillo, 16(2), 182199. http://revistas.ucv.edu.pe/index.php/R_PSI/ article/download/258/152

Borrás, T., Reynaldo, A., \& López, M. (2017). Adolescentes: razones para su atención. Correo Científico Médico, 21(3), 858-875. http://scielo. sld.cu/pdf/ccm/v21n3/ccm20317.pdf

Cortés, M. T., Petra, I., Acosta, E., Reynaga, J., Fouilloux, M., García, R., \& Piedra, E. (2017). Desarrollo y crecimiento personal. Construcción y validación de un instrumento para evaluar esta competencia en alumnos de medicina. Revista de La Fundación Educación Médica, 20(2), 65. http://scielo.isciii.es/pdf/fem/v20n2/2014-9832fem-20-2-65.pdf

Dewi, A. K., Agustin, R. W., \& Satwika, P. A. (2018). The Relationship between Emotion Maturity and Social Adjustment with Migrant Employees' Psychological Well-being at PT. Pelabuhan Samudera Palaran Samarinda. Advances in Social Science, Education and Humanities Research (ASSEHR), 127(1), 87-90. https://doi. org/10.2991/icaaip-17.2018.18

Diario Peru21. (2018). Cifras de salud mental en Perú: Menores ocupan el $70 \%$ de atenciones. Peru21. https://peru21.pe/peru/cifras-saludmental-peru-menores-ocupan-70-atencionesinfografia-394376

Díaz, D., Rodríguez-Carvajal, R., Blanco, A., MorenoJiménez, B., Gallardo, I., Valle, C., \& Van, D. (2006). Adaptación española de las escalas de bienestar psicológico de Ryff. Psicothema, 18(3), 572-577. http://www.psicothema.com/pdf/3255. pdf

Fossas, A. (2019). Psychological Maturity Predicts Different Forms of Happiness. Journal of Happiness Studies, 20(6), 1933-1952. https:// doi.org/10.1007/s10902-018-0033-9 
Freire Rodríguez, C. (2014). El bienestar psicológico en los estudiantes universitarios: operativización del constructo y análisis de su relación con las estrategias de afrontamiento [Universidad de la Coruña]. https://ruc.udc.es/dspace/bitstream/ handle/2183/13815/FreireRodriguez_Carlos_ TD_2014.pdf?sequence $=6 \&$ isAllowed $=\bar{y}$

Garcia-Landete, J. (2012). Psicología positiva, bienestar y calidad de vida. En-Claves Del Pensamiento, 8(16), 13-29. http://www. scielo.org.mx/pdf/enclav/v8n16/1870-879Xenclav-8-16-00013.pdf

García, E., Muñoz, N., Ramírez, K., \& Mérida, R. (2015). Conductas de riesgo en los adolescentes. Revista Cubana de Medicina Militar, 44(2), 218229. http://scielo.sld.cu/pdf/mil/v44n2/mil10215. pdf

Gaxiola-Villa, E. (2014). Bienestar psicológico y desgaste profesional [DP] en amas de casa docentes universitarias. Journal of Behavior, Health \& Social, 6(1), 79-88. https://doi. org/10.5460/jbhsi.v6.1.47605

González-Fuentes, M. B., \& Andrade, P. (2013). Autoaceptación como factor de riesgo para el intento de suicidio en adolescentes. Salud \& Sociedad, 4(1), 26-35. http://pepsic.bvsalud.org/pdf/salsoc/ v4n1/4n1a02.pdf

Hernandez, R., Fernández, C., \& Baptista, P. (2014). Metodología de la investigación (6th ed.). McGrawHill.

Joy, M., \& Mathew, A. (2018). Emotional Maturity and General Well-Being of Adolescents. IOSR Journal Of Pharmacy, 8(5), 2250-3013. https:// www.researchgate.net/publication/333918195 Emotional_Maturity_and_General_Well-Being_ of_Adolescents

Kern, M., Waters, L., Adler, A., \& White, M. (2015). A multidimensional approach to measuring wellbeing in students: Application of the PERMA framework. The Journal of Positive Psychology, 10(3), 262-271. https://psycnet.apa.org/doi/10.1 080/17439760.2014.936962

McCrae, R. R. (2002). The maturation of personality psychology: Adult personality development and psychological well-being. Journal of Research in Personality, 36(4), 307-317. https://doi. org/10.1016/S0092-6566(02)00011-9

Ministerio de Salud. (2017). Situación de salud de los adolescentes y jóvenes en el Perú. In MINSA. http://bvs.minsa.gob.pe/local/MINSA/4143.pdf

Navarro-Loli, J. S., Moscoso, M., \& CalderónDe La Cruz, G. (2017). La investigación de la depresión en adolescentes en el Perú: una revisión sistemática. Liberabit: Revista
Peruana de Psicología, 23(1), 57-74. https://doi. org/10.24265/liberabit.2017.v23n1.04

Orgnización Mundial de la Salud. (2017). Cada año fallecen más de 1,2 millones de adolescentes por causas que, en su mayor parte, podrían evitarse. OMS; World Health Organization. http://www. who.int/mediacentre/news/releases/2017/yearlyadolescent-deaths/es/

Páramo, M. de los Á., Straniero, C. M., García, C. S., Torrecilla, N. M., \& Gómez, E. E. (2012). Bienestar psicológico, estilos de personalidad y objetivos de vida en estudiantes universitarios. Pensamiento Psicológico, 10(1), 7-21. http:// www.scielo.org.co/pdf/pepsi/v10n1/v10n1a01.pdf

Perales, A., Izaguirre, M., Sanchez, E., Barahona, L., Martina, M., Amemiya, I., Delgado, A., Dominguez, I., Pinto, M., \& Padilla, A. (2019). Salud mental en estudiantes de pregrado de la Facultad de Medicina de la Universidad Nacional Mayor de San Marcos. Anales de La Facultad de Medicina, 80(4), 443-450. http://www.scielo.org. pe/pdf/afm/v80n4/a06v80n4.pdf

Rodrigo, J., \& Andreu, J. (2017). Evaluación Psicológica De La Madurez Psicosocial En Adolescentes. Psicopatología Clínica, Legal y Forense, 17, 14-31. https://dialnet.unirioja.es/ descarga/articulo/6674241.pdf

Vera, V. (2016). Propiedades Psicométricas del Cuestionario de Madurez Psicológica (PSYMAS) en Líderes Juveniles de las diferentes sedes de la Universidad Cesar Vallejo. [Universidad Cesar Vallejo]. https://repositorio.ucv.edu.pe/ bitstream/handle/20.500.12692/194/vera_ cv.pdf?sequence $=1$ \&isAllowed $=y$

Zacarés, J., \& Serra, E. (1996). Creencias sobre la madurez psicológica y desarrollo adulto. Anales de Psicología, 12(1), 41-60. https://www.um.es/ analesps/v12/v12_1/04-12-1.pdf

Recibido: 21/02/2021

Aceptado: 23/05/2021 